Arnaldo L. Colombo Thais Guimarães Teresa Sukienik Alessandro C. Pasqualotto

Ricardo Andreotti Flavio Queiroz-Telles Simone A. Nouér Marcio Nucci

\section{Erratum to: Prognostic factors and historical trends in the epidemiology of candidemia in critically ill patients: an analysis of five multicenter studies sequentially conducted over a 9-year period}

Published online: 6 November 2014

(C) Springer-Verlag Berlin Heidelberg and ESICM 2014

The online version of the original article can be found under doi:10.1007/s00134-014-3400-y.

\section{A. L. Colombo · R. Andreotti}

Escola Paulista de Medicina,

Universidade Federal de São Paulo,

São Paulo, Brazil

T. Guimarães

Hospital do Servidor Público Estadual de São Paulo,

São Paulo, Brazil

T. Sukienik

Irmandade da Santa Casa de Misericórdia de Porto Alegre,

Porto Alegre, Brazil

A. C. Pasqualotto

Universidade Federal de Ciências da Saúde de Porto Alegre,

Porto Alegre, Brazil

F. Queiroz-Telles

Universidade Federal do Paraná, Curitiba, Brazil

S. A. Nouér · M. Nucci

Universidade Federal do Rio de Janeiro,

Rio de Janeiro, Brazil

A. L. Colombo (๘)

Rua Pedro de Toledo, 669, 04039-032 São Paulo,

SP, Brazil

e-mail: arnaldolcolombo@gmail.com

Tel.: 55-11-55764985

\section{Erratum to: Intensive Care Med (2014) \\ 40:1489-1498 \\ DOI 10.1007/s00134-014-3400-y}

The authors would like to add to their article a short statement regarding their potential conflicts of interest not specifically related to the funding of this particular publication. The potential conflicts of interest are as follows:

Arnaldo Lopes Colombo

Consulting: Pfizer, Gilead, MSD, United Medical; medical education programs: Pfizer, Astellas, MSD, United Medical; research grants: Pfizer, MSD, Luminex.

Alessandro Pasqualotto

Consulting: Pfizer, Gilead, Astellas, MSD; medical education programs: Pfizer, Gilead, Astellas, MSD; research grants: Pfizer, MSD, Gilead.

Marcio Nucci

Consulting: Pfizer, Gilead, Astellas, Merck; medical education programs: Pfizer, Gilead, Astellas, Merck; research grants: Pfizer, Merck.

Flavio Queiroz-Telles

Consulting: Merck, United Medical, Teva; medical education programs: Pfizer, Teva, United; research grants: Pfizer, Astellas, Merck.

No conflicts of interest

Thais Guimarães, Teresa Sukienik, Simone Nóuer, Ricardo Andreotti. 